\title{
Focal myocardial fibrosis assessed by late gadolinium enhancement cardiovascular magnetic resonance in children and adolescents with dilated cardiomyopathy
}

Heiner Latus ${ }^{1 *}$, Kerstin Gummel ${ }^{1}$, Karin Klingel ${ }^{2}$, Axel Moysich ${ }^{1}$, Markus Khalil ${ }^{1}$, Nona Mazhari ${ }^{1}$, Juergen Bauer ${ }^{1}$, Reinhard Kandolf ${ }^{2}$, Dietmar Schranz ${ }^{1}$ and Christian Apitz ${ }^{1}$

\begin{abstract}
Background: Different patterns of late gadolinium enhancement (LGE) including mid-wall fibrosis using cardiovascular magnetic resonance (CMR) have been reported in adult patients presenting with non-ischemic dilated cardiomyopathy (DCM). In these studies, LGE was associated with pronounced LV remodelling and predicted adverse cardiac outcomes. Accordingly, the purpose of our study was to determine the presence and patterns of LGE in children and adolescents with DCM.

Methods: Patients $<18$ years of age presenting with severe congestive heart failure who were admitted for evaluation of heart transplantation at our centre underwent CMR examination which consisted of ventricular functional analysis and assessment of LGE for detection of myocardial fibrosis. Ischemic DCM was excluded by coronary angiography, and right ventricular endomyocardial biopsies ruled out acute myocarditis.

Results: Thirty-one patients (mean age $2.1 \pm 4.2$ years) with severe LV dilatation (mean indexed LVEDV $136 \pm 48 \mathrm{ml} / \mathrm{m}^{2}$ ) and LV dysfunction (mean LV-EF $23 \pm 8 \%$ ) were examined. LGE was detected in 5 of the 31 patients (16\%) appearing in various patterns characterized as mid-wall $(n=1)$, focal patchy $(n=1)$, RV insertion site $(n=1)$ and transmural $(n=2)$. Based on histopathological analysis, 4 of the 5 LGE positive patients had lymphocytic myocarditis, whereas one patient was diagnosed with idiopathic DCM.

Conclusions: In children and adolescents with DCM, focal histologically proven myocardial fibrosis is rarely detected by LGE CMR despite marked LV dilatation and severely depressed LV function. LGE occurred in various patterns and mostly in patients with inflammatory cardiomyopathy. It remains unclear whether myocardial fibrosis in childhood DCM reflects different endogenous repair mechanisms that enable favourable reverse remodelling. Larger trials are needed to assess the prognostic implications of LGE in childhood DCM.
\end{abstract}

Keywords: Childhood dilated cardiomyopathy, Myocardial fibrosis, Late gadolinium enhancement, Reverse ventricular remodelling

\footnotetext{
* Correspondence: heiner.latus@googlemail.com

${ }^{1}$ Pediatric Heart Centre, University Children's Hospital Giessen, Feulgenstr. 12.

D-35392 Giessen, Germany

Full list of author information is available at the end of the article
} 


\section{Background}

Dilated cardiomyopathy (DCM) is the most common form of cardiomyopathy in children and is characterized by a dilated and globally hypocontractile left ventricle. Childhood DCM is a heterogenous disease associated with high morbidity and mortality [1-3], especially in patients with idiopathic DCM [4]. Patients frequently develop severe congestive heart failure. If pharmacological treatment fails, patients are listed for cardiac transplantation (HTx) or must receive mechanical circulatory support. However, the clinical course can be variable and some patients remain stable or even show reverse remodelling with normalization of LV dimensions and function $[5,6]$. Although specific risk factors for adverse outcome have been found in children with DCM [2, 4, 7, 8], additional parameters for reliable risk stratification and prediction of a reverse ventricular remodelling are required to guide management of these patients.

Cardiovascular magnetic resonance (CMR) has gained an important role in the diagnosis of patients presenting with systolic heart failure. Late gadolinium enhancement (LGE) imaging enables identification and quantification of focal macroscopic myocardial fibrosis. In adult patients with non-ischemic DCM, various studies demonstrated not only a diagnostic but also prognostic role of LGE CMR [9-12]. In these studies, different patterns of local myocardial fibrosis including the typical pattern of "mid-wall" fibrosis assessed by LGE have been reported in up to $50 \%$ of adult patients with DCM [9-13].

CMR assessment of myocardial fibrosis has been reported to be useful in patients with congenital heart disease $[14,15]$ and cardiomyopathies [16], however, data about LGE imaging in children is limited. The purpose of our study was to determine whether biopsy-proven myocardial fibrosis can be assessed by LGE in children with DCM and whether its occurrence is related to the cause of DCM and in what pattern myocardial fibrosis occurs. Furthermore, the impact of myocardial fibrosis on reverse ventricular remodelling should be evaluated.

\section{Methods}

\section{Study population}

Patients under 18 years of age presenting with congestive heart failure who were admitted for evaluation of cardiac transplantation at our centre underwent CMR as part of our routine clinical examination. The diagnosis of DCM was made according to current guidelines: patients with a dilated left ventricle, defined by left ventricular end-diastolic dimension with a z-score of greater than 2 and an ejection fraction below a z-score of -2 were included in the study. Patients with congenital cardiac disease including abnormal origin of the coronary arteries or Kawasaki disease, neuromuscular or immunologic disease, familial cardiomyopathy, endocrine disorders, metabolic or mitochondrial disease and clinical evidence of acute myocarditis (based on the results of the histopathological/ immunohistological analysis of the endomyocardial biopsies) were excluded.

Clinical data were retrospectively obtained from hospital medical records including date of birth, gender, cause of DCM and age at CMR evaluation. Findings of the CMR studies were extracted from the routine clinical reports. Laboratory findings of B-type natriuretic peptide (BNP) and troponin I (TNI) at initial admission were also assessed. Clinical follow-up data of the patients included information about further treatment (i.e. medical heart failure therapy, pulmonary artery banding, left ventricular mechanical support, cardiac transplantation) and survival. Patients who were treated by medical heart failure therapy, follow-up data on echocardiographic data (LV dimension and function) were also assessed for the evaluation of reverse ventricular remodelling (defined as normalisation of LV size and function, i.e. z-score of LVEDD and fractional shortening between +2 to -2 [5]). The study protocol was approved by the local ethics committee and all patients or parents of the patients gave written informed consent for participation in the study.

\section{Cardiac catheterization protocol, histopathological/ immunohistological analysis of endomyocardial biopsies and detection of viral genomes}

Cardiac catheterization was performed in all patients. Endomyocardial biopsies were performed in 30 of the 31 patients. In each patient two to three biopsies were taken from the RV interventricular septum using a transcatheter bioptome. In one patient there was no myocardial biopsy available, because myocardial perforation occurred at the first attempt of biopsy sampling with subsequent need for emergency surgery.

Histopathological analysis was performed as previously described [17, 18]. Endomyocardial biopsies were stained with Masson's trichrome as well as Giemsa and examined by light microscopy. Inflammatory cells were quantified with immunohistochemical staining using CD3 ( $\mathrm{T}$ cells), CD68 (macrophages) and HLA-DR- $\alpha$ to assess HLA class II expression in professional antigenpresenting immune cells. Nested (RT-) polymerase chain reaction was performed for the detection of enteroviruses (including coxsackieviruses group $\mathrm{A}$ and $\mathrm{B}$ and echoviruses), parvovirus B19 (PVB19), adenoviruses, human cytomegalovirus, Epstein-Barr virus, and human herpes virus type 6 (HHV6). The heart was considered to be inflamed after immunohistochemical detection of focal or diffuse mononuclear infiltrates with $>14$ leukocytes per $1 \mathrm{~mm}^{2}\left(\mathrm{CD}^{+} \mathrm{T}\right.$ lymphocytes and/or $\mathrm{CD}^{+} 8^{+}$macrophages) in the myocardium, in addition to enhanced expression of HLA class II molecules. 


\section{CMR protocol}

All CMR studies were performed on a 3-T system (Verio, Siemens, Erlangen, Germany). Images were acquired with two sixteen-elements phased array coils. Sedation was applied in most of the patients.

\section{Cine CMR}

Images were acquired in supine position with two sixteenelements phased array coils. Sedation was applied in younger patients when considered necessary. The CMR protocol included a stack of short-axis slices from the base of the heart to the apex using cine steady-state free precession (SSFP) with breath-hold or gradient echo (GE) sequences in free-breathing technique when patients were sedated. Data acquired during breath-hold were assessed with the following sequence parameters: TR $48 \mathrm{~ms}$, TE $1.5 \mathrm{~ms}$, flip angle $60^{\circ}$, slice thickness $6 \mathrm{~mm}$, in plane image resolution $1.3 \mathrm{~mm} \times 1.3 \mathrm{~mm} \times 6.0 \mathrm{~mm}$, temporal resolution $25-40$ phases. In measurements during free-breathing, the sequence parameters were as follows: TR $56 \mathrm{~ms}$, TE $2.5 \mathrm{~ms}$, flip angle $12^{\circ}$, slice thickness $5 \mathrm{~mm}$, in plane image resolution $1.4 \mathrm{~mm} \times 1.4 \mathrm{~mm} \times 5.0 \mathrm{~mm}$. End-diastolic (maximal) and end-systolic (minimal) volumes, stroke volumes (SV) and ejection fractions (EF) for the RV and LV were calculated by dedicated software (ARGUS, Siemens, Erlangen, Germany) after the RV and LV endocardial borders were traced manually at end-systole and end-diastole. All parameters were adjusted to body surface area (BSA).

\section{Late gadolinium enhancement CMR}

Before administration of gadolinium, renal function parameters, i.e. glomerular filtration rate, as well as creatinine levels were assessed to exclude renal dysfunction. LGE was assessed after intravenous injection of gadopentetate dimeglumine (Magnevist, Bayer, Leverkusen, Germany) at a dose of $0.15 \mathrm{mmol} / \mathrm{kg}$ of body weight by using a two-dimensional inversion-recovery segmented gradient echo CMR sequence in the cardiac short and long-axis planes (slice thickness $5 \mathrm{~mm}$, field of view $300 \mathrm{~mm}$ ). The inversion time was adjusted for optimal suppression of signal from normal myocardium and the images were obtained within 5-10 min after injection (inversion time approximately 250-350 ms). All LGE images were interpreted accordingly to the American Heart Association 17-segment model. The patterns of LGE were classified as subendocardial-based, transmural, mid-wall striae, mid-wall patchy, subepicardial, RV insertion point and diffuse. Extent of LGE was assessed using certified software $\left(\mathrm{cmr}^{42}\right.$, Circle Cardiovascular Imaging Inc., Calgary, Canada).

\section{Statistical analysis}

Continuous variables are presented as mean with standard deviation or median and range, as appropriate. Correlations were tested using linear regression analysis. Analysis was performed using GraphPad statistical software package (San Diego, California, USA). A $p$ value $\leq 0.05$ was considered statistically significant.

\section{Results}

\section{Patient population}

Between 01/2009 and 05/2014 thirty-one patients (17 females, median age 7.0 (1-203) months, median weight $6.6(3-57) \mathrm{kg}$ ) were included in the study. The majority of patients $(n=21,68 \%)$ were less than one year of age (Table 1). Associated congenital heart disease included an atrial septal defect in one patient. On admission, 16

\section{Table 1 Patient characteristics}

\begin{tabular}{|c|c|}
\hline Variable & Value \\
\hline Patients, $n$ & 31 \\
\hline Male/Female & $14 / 17$ \\
\hline Height, cm & $65(53-161)$ \\
\hline Weight, kg & $6.6(3-57)$ \\
\hline $\mathrm{BSA}, \mathrm{m}^{2}$ & $0.46 \pm 0.33$ \\
\hline Age at study, months & $7(1-203)$ \\
\hline Age < 4 weeks, n (\%) & $3(10)$ \\
\hline Age 1-24 months, n (\%) & $21(68)$ \\
\hline Age 2-12 years, n (\%) & $5(16)$ \\
\hline Age $12-18$ years, $n(\%)$ & $2(6)$ \\
\hline \multicolumn{2}{|l|}{ Cause of DCM } \\
\hline Unknown, n (\%) & $15(48)$ \\
\hline Chronic lymphocytic myocarditis, n (\%) & $16(52)$ \\
\hline Detection of virus by endomyocardial biopsy, n (\%) & $11(35)$ \\
\hline HHV6, n & 6 \\
\hline PVB19, n & 3 \\
\hline Enteroviruses, $\mathrm{n}$ & 2 \\
\hline \multicolumn{2}{|l|}{ Blood testing } \\
\hline Troponin positive, n (\%) & $17(55)$ \\
\hline Tropnin, $\mu \mathrm{g} / \mathrm{l}$ & $0.18 \pm 0.28$ \\
\hline $\mathrm{BNP}, \mathrm{pg} / \mathrm{ml}$ & $1900 \pm 2019$ \\
\hline NYHA-class, I/II/III/IV, n & $0 / 2 / 13 / 16$ \\
\hline \multicolumn{2}{|l|}{ Medication } \\
\hline ACE-I/ARB, n (\%) & $24(77)$ \\
\hline Beta-blocker, n (\%) & $24(77)$ \\
\hline Diuretics, n (\%) & $27(87)$ \\
\hline Digoxin, n (\%) & $13(42)$ \\
\hline Antiarrhytmic, n (\%) & $5(16)$ \\
\hline Inotropcis, n (\%) & $16(52)$ \\
\hline
\end{tabular}

DCM dilated cardiomyopathy, BSA Body surface area, PVB19 parvovirus B19, HHV6, human herpes virus type 6, BNP b-type natriuretic peptide, NYHA New York Heart Association, ACE-I angiotensin-converting inhibitors, ARB angiotensin receptor blocker; Data are presented as mean standard deviation (SD) or median and range, as appropriate 
patients were in NYHA/Ross class IV, 13 patients in class III and 2 patients in class II. Mitral valve regurgitation on echocardiography was ${ }^{\circ} 1$ in 16 patients, ${ }^{\circ} 2$ in 12 patients and ${ }^{\circ} 3$ in 3 patients. Mean levels of BNP were $1900 \pm 2019 \mathrm{pg} / \mathrm{ml}$ and mean TNI levels were $0.18 \pm$ $0.28 \mu \mathrm{g} / \mathrm{l}$.

\section{Endomyocardial biopsies}

According to the results of the histopathological/ immunohistological analysis of the endomyocardial biopsies, 15 patients (48\%) revealed DCM without and 16 patients $(52 \%)$ with inflammation (DCMi, inflammatory cardiomyopathy) corresponding to the diagnosis of chronic myocarditis (Table 1). Positive viral genome testing was reported in 11 patients with detection of HHV6 in 6, PVB in 3 and enteroviruses in 2 patients. Histopathological analysis was not available in one patient (who showed no signs of acute myocarditis and had negative viral serology testing)

\section{CMR findings}

Mean heart rate at CMR was $115 \pm 22 \mathrm{bpm}$. Mean indexed (i) LVEDV was $136 \pm 48 \mathrm{ml} / \mathrm{m}^{2}$ and mean LVESVi $106 \pm$ $42 \mathrm{ml} / \mathrm{m}^{2}$ resulting in an LVSVi of $30 \pm 14 \mathrm{ml} / \mathrm{m}^{2}$ (Table 2). Mean LV ejection fraction (EF) was $23 \pm 8 \%$. Mean RVEDVi was $65 \pm 36 \mathrm{ml} / \mathrm{m}^{2}$, mean RVESVi $36 \pm 30 \mathrm{ml} / \mathrm{m}^{2}$ and RVSVi $29 \pm 11 \mathrm{ml} / \mathrm{m}^{2}$. Mean RV-EF was $50 \pm 13 \%$ but ranging from 12 to $66 \%$.

LGE was detected in 5 of the 31 patients (16\%) appearing in various patterns characterized as focal patchy (1 patient, aged 12 months with lymphocytic myocarditis), transmural (2 patients, aged 5 and 7 months, both lymphocytic myocarditis), RV insertion fibrosis (1 patient aged 1 month with lymphocytic myocarditis) and classic 'mid-wall'

Table 2 CMR findings

\begin{tabular}{lc}
\hline Variable & Value \\
\hline Heart rate, bpm & $115 \pm 22$ \\
LVEDVi, ml/m² & $136 \pm 48$ \\
LVESVi, ml/m ${ }^{2}$ & $106 \pm 42$ \\
LVSVi, $\mathrm{ml} / \mathrm{m}^{2}$ & $30 \pm 14$ \\
LVEF, \% & $23 \pm 8$ \\
RVEDVi, ml/m² & $65 \pm 36$ \\
RVESVi, ml/m & $36 \pm 30$ \\
RVSVi, $\mathrm{ml} / \mathrm{m}^{2}$ & $29 \pm 11$ \\
RVEF, \% & $50 \pm 13$ \\
LGE positive, n (\%) & $5(16)$ \\
LGE, \% of LV mass & $6.1 \pm 5.3$
\end{tabular}

bpm, beats per minute; LV, left ventricle; RV, right ventricle; i, indexed; EDV, enddiastolic volume; ESV, endsystolic volume; SV, stroke volume;EF, ejection fraction; LGE, late gadolinium enhancement; Data are presented as mean standard deviation (SD)
(1 patient, aged 16 years, idiopathic DCM) (Figs. 1 and 2). The mean extent of LGE of moycardial mass was $6.1 \pm 5.3 \%$ (range from 1.7 to $14.7 \%$ ).

\section{Correlations}

Levels of troponin I were related to the degree of LV dysfunction $(r=-0.39, p=0.03)$ and showed a trend with increasing LV diameter $(r=0.33, p=0.07)$ (Fig. 3). Levels of B-type natriuretic peptide correlated significantly with LVEDVi $(\mathrm{r}=0.36, \mathrm{p}=0.04)$ and LVESVi $(\mathrm{r}=0.44, p=0.02)$ but not with LV-EF $(r=-0.24, p=0.19)$. RV parameters were not related to BNP levels. Significant correlations were found between LVEDVi and RV-EF $(r=-0.44$, $p=0.01)$, whereas LVEDVi showed no relationship with RVEDVi $(\mathrm{r}=0.20, p=0.24)$. No relationship was found between LV-EF and RV-EF $(r=0.03, p=0.88)$.

\section{Follow-up and occurrence of reverse ventricular remodelling}

Of the 26 LGE negative patients 13 underwent pulmonary artery banding (PAB) (Fig. 4) as a rescue therapy combined with medical heart failure therapy as previously reported by our group [19]. This intervention was offered for patients with preserved right ventricular function and informed consent was obtained from the parents for this compassionate use treatment. Another 6 patients underwent cardiac transplantation while 7 patients received only medical heart failure therapy. Of these latter patients, one patient showed spontaneous reverse remodelling, four patients were clinically stable with unchanged levels of LVEDD values and LV function while one patient died and another one was lost to follow-up. Of the five LGE positive patients, none died or had to undergo mechanical circulatory support. One of the patients with transmural LGE underwent mitral valve replacement due to progressive severe mitral valve insufficiency (probably related to myocardial scarring) leading to further atrial and ventricular enlargement while the other patient underwent cardiac transplantation. In the two other patients (one with focal patchy and one with RV insertion fibrosis) cardiac dimensions and function recovered to normal values after 3 and 22 months indicating spontaneous reverse remodelling. The 16 yearold patient with mid-wall fibrosis declined implantation of a cardioverter-defibrillator due to episodes of ventricular tachycardia while clinical status and cardiac dimensions remained stable.

\section{Discussion}

This study demonstrated that in young children and adolescents with dilated cardiomyopathy

(1) The detection of histologically proven focal myocardial fibrosis by LGE is uncommon (found 


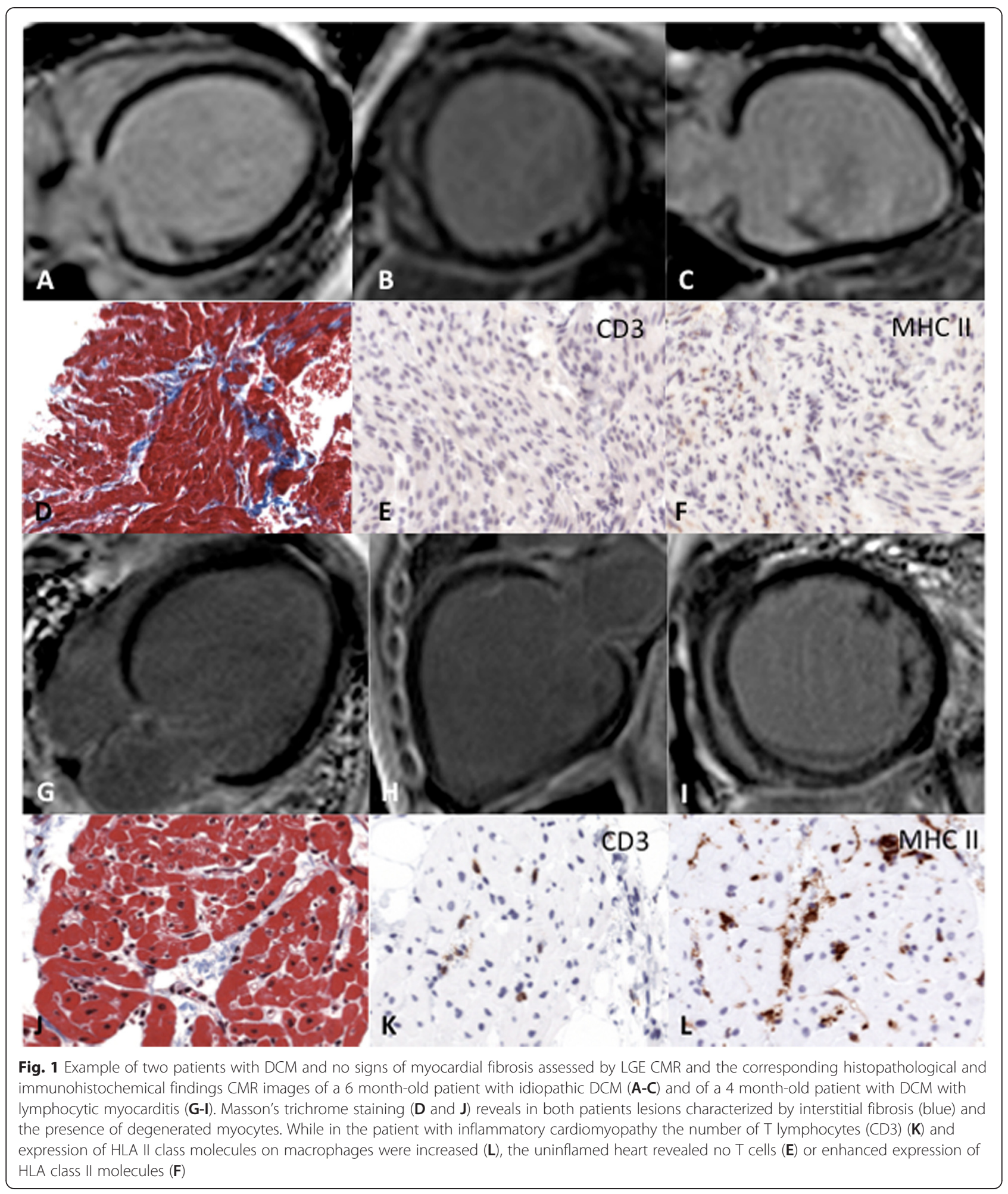

only in 16\%) although LV dilatation was marked and LV function severely depressed,

(2) The majority of LGE positive patients had chronic myocarditis suggesting a relation between histological signs of inflammation and LGE,
(3) Various patterns of LGE were found with transmural type of LGE being detected in two of the 5 patients, which is not typical for non-ischemic forms of DCM and might reflect embolic myocardial infarction. 


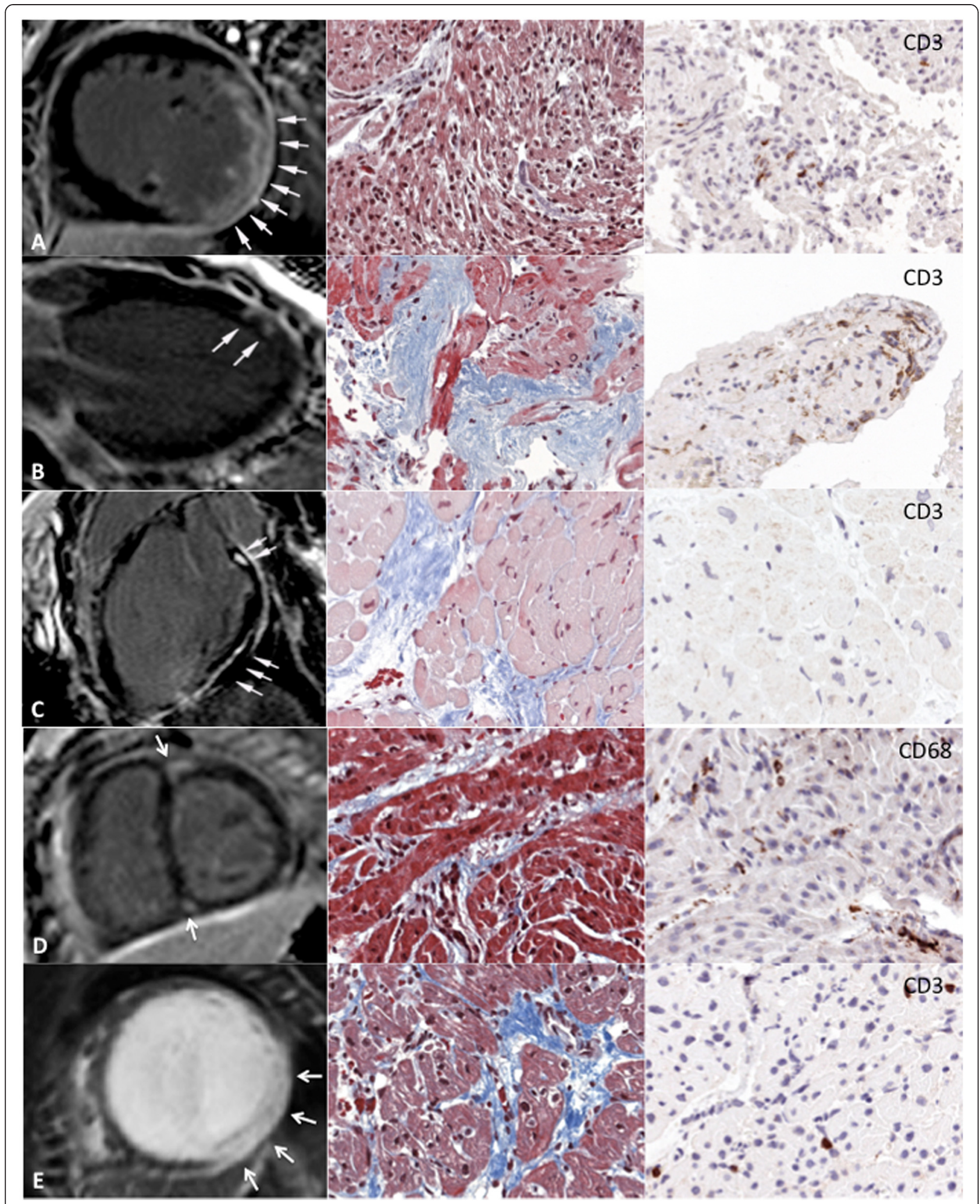

Fig. 2 (See legend on next page.) 
(See figure on previous page.)

Fig. 2 Results of CMR (left column) and histopathology/immunohistology of the five patients in whom LGE was detected. Various patterns of LGE were found (transmural LGE in A and E; focal patchy LGE in C; midwall LGE in C; RV insertion LGE in D). The column in the middle shows the Masson's trichrome staining illustrating the presence of fibrosis (blue) while the right column displays immunohistochemical analysis reveals detection of T cells (CD3) or macrophages (CD68). Inflammatory cardiomyopathy was found in four patients (A,B,D,E) whereas the heart of patient C showed no signs of inflammation

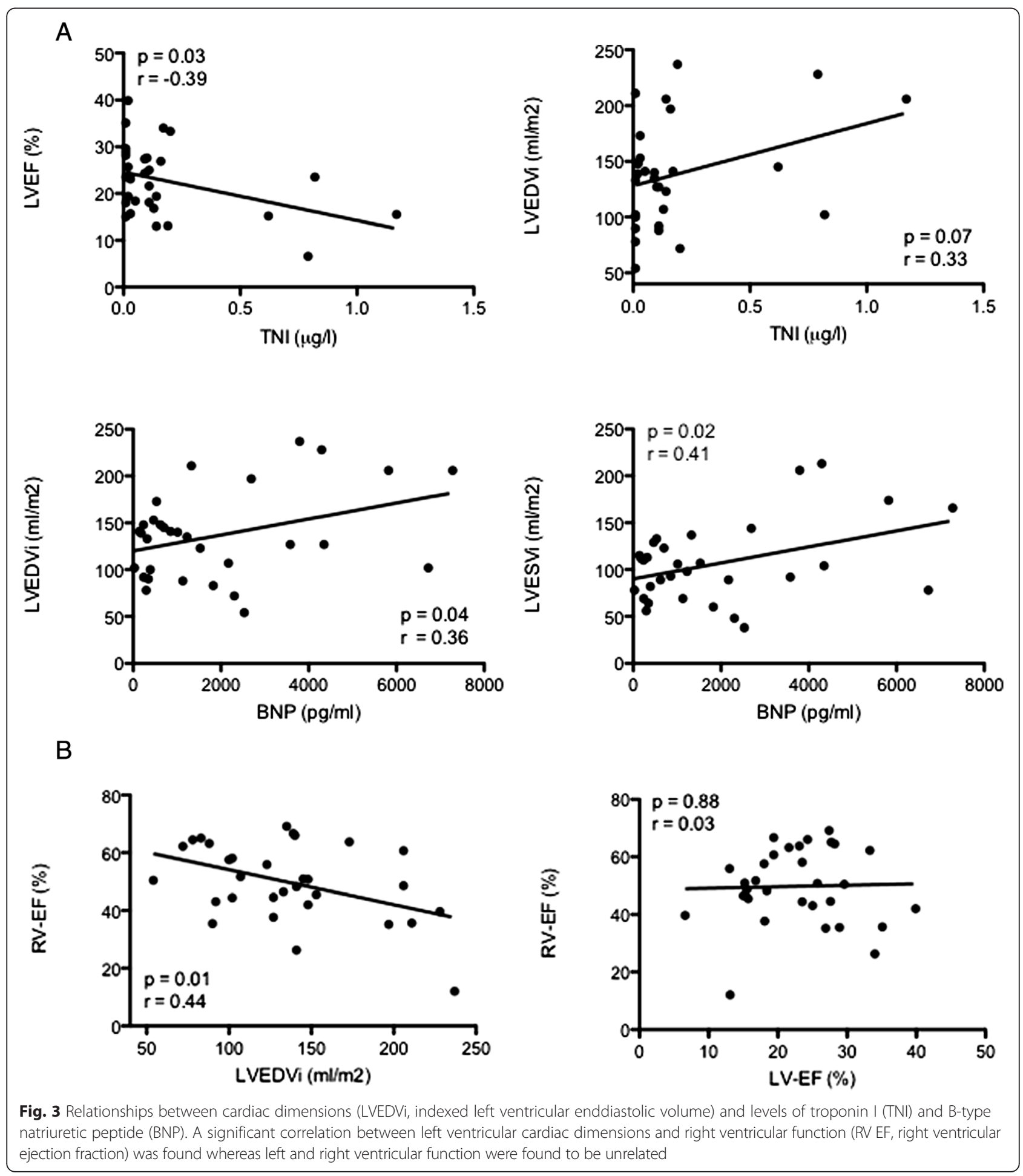




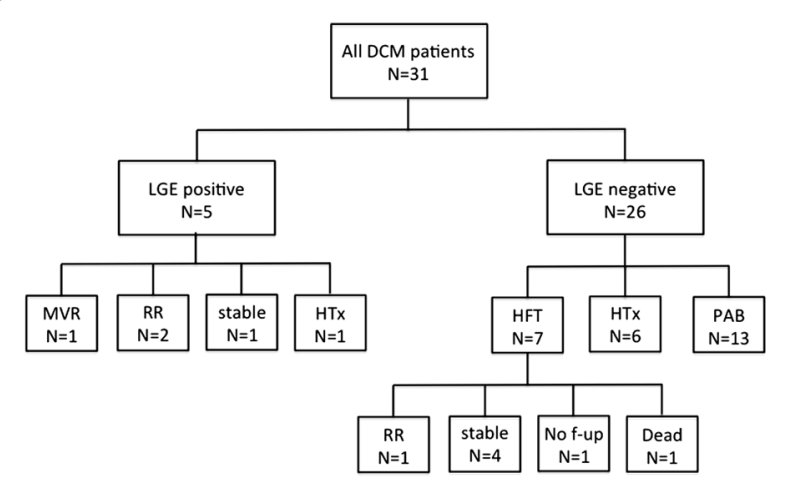

Fig. 4 Follow-up including outcome and occurrence of reverse remodelling in the study population. DCM, dilated cardiomyopathy; $L G E$, late gadolinium enhancement; $M V R$, mitral valve replacement; $R R$ reverse remodelling; $H F T$, medical heart failure therapy; $H T X$, cardiac transplantation; $P A B$, pulmonary artery banding; $f$-up, follow-up

The findings of our study are in contrast to previously reported CMR findings in adult patients with nonischemic DCM where LGE was detected more frequently (in up to $50 \%$ of patients) and was associated with larger left ventricles, a higher degree of impaired function, elevated biomarkers and arrhythmias resulting in worse overall outcome [9-12, 20]. Accordingly, the presence of focal LGE in non-ischemic DCM in adults is considered to be associated with contractile impairment that is thought to reflect the transition from compensated to decompensated state representing the endpoint of adverse remodelling with irreversible ventricular failure [9]. As the severity of LV dilatation and functional impairment in our study group was comparable to that reported in series of adult patients, the low rate of macroscopic fibrosis detected by LGE in our study is unexpected and the underlying reason for this finding entirely unknown.

In general, limited data exists about the pathogenesis of myocardial fibrosis in neonates, children and young adolescents with all types of cardiomyopathies including its assessment by LGE [16, 21-23]. Grosse-Wortmann and colleagues [24] reported 11 children and adolescents with DCM of various etiologies but found myocardial fibrosis by LGE only in one patient. Although the severity of LV dilatation and dysfunction was more pronounced in our study group, the prevalence of myocardial fibrosis by LGE did not increase. Furthermore, as reported by this group, RV dimensions and function differed widely among the patients illustrating that childhood DCM can present as a biventricular disease although no relationship between RV and LV dimensions was detected. However, the severity of LV dilatation negatively affected RV function probably due to adverse ventricular-ventricular interactions while BNP levels were solely related to LV dimensions.
Specific patterns of LGE have been reported in patients with ischemic and non-ischemic DCM linking the type of LGE to a certain form of cardiomyopathy. In nonischemic DCM mid-wall striae of LGE is known to be the typical finding, although various patterns such as focal patchy, subepicardial and diffuse LGE have also been described [9-12, 25, 26]. Interestingly, the only patient in our study with mid-wall LGE had idiopathic DCM and was the oldest patient in the study cohort (17 years of age) while a midwall focal patchy pattern of LGE and RV insertion site fibrosis were seen in one patient with lymphocytic myocarditis, respectively. It has been described that myocarditic infiltrations can persist after the acute phase of myocarditis, but tend to regress over time during healing $[17,27-29]$. Conversely, a transmural pattern of fibrosis is not typically for myocarditis or idiopathic DCM, rather than for ischemic heart disease, but was found in two patients with myocarditis. These scars could be related to an embolic event that subsequently caused myocardial infarction or might be associated with interstitial expansion due to inflammation and/or edema as these patients were diagnosed for inflammatory cardiomyopathy. However, discrimination of ischemic from non-ischemic lesions is crucial in these patients and thus might influence therapeutic strategy and prognosis.

The underlying mechanisms for the development of fibrotic changes in non-ischemic cardiomyopathies are related to different processes. Myocardial injury can be the result of different pathogens and toxins causing inflammation, individual genetic susceptibility, abnormal modulation of the immune system, permanent adrenergic activation and metabolic dysregulation [30-32]. Furthermore, increased wall stress due to LV enlargement can lead to microvascular ischemia with subsequent myocyte necrosis which might explain why troponin levels were found to be related to LV dimension and function in our population. The resulting focal areas of replacement fibrosis are thought to be the basis for the detection of LGE by CMR although LGE can also be the result of other forms of interstitial expansion. Although good agreement between areas of LGE and the pathological location of macroscopic fibrosis on autopsy has been reported [10,11], the study by Schalla and colleagues showed that the presence of focal LGE in adult DCM patients was more related to microscopic findings of inflammation rather than fibrosis in endomyocardial biopsy [33]. This might explain why the majority (4 of the 5 patients) of our LGE positive patients were found to have lymphocytic myocarditis. Otherwise, the low detection rate of LGE in our population does not imply that myocardial fibrosis is absent in childhood DCM. Indeed, only a few histopathological studies exist about childhood DCM, but those also revealed fibrosis as a cardinal feature $[34,35]$. This is in accordance with our findings, where analysis of endomyocardial biopsies 
revealed a substantial degree of fibrosis in endomyocardial biopsies, both in patients with and without LGE on CMR.

This suggests that the possible pathogenic factors and mechanisms leading to the phenotype of LV dilatation and dysfunction considerably vary in younger patients. Although speculative, the low incidence of myocardial fibrosis assessed by CMR might be related to differences in myocardial remodelling in DCM between children and adults. Nishikawa et al. analysed endomyocardial biopsies and found differences in the distribution of microscopic patterns of fibrosis, revealing a higher myocarditic index and a higher incidence of bizarre myocardial hypertrophy as well as discrete ultrastructural differences between adults and children with DCM [34, 36] suggesting that factors and mechanisms causing myocardial damage may differ between children and adults. Furthermore, differences in myocardial matrix structure and function [37] as well as in adrenergic receptor stimulation [38] have been described, which might have an impact on response to medical therapy thereby influencing ventricular remodelling and prognosis.

Decision making whether cardiac transplantation is needed in the individual child with DCM is crucial but is hampered by a substantial rate of spontaneous recovery of ventricular dimension and function that has been described both in adult and childhood DCM $[3,5,6,39,40]$. A recent multicentre study by Everitt et al. demonstrated a 2-year cumulative incidence of recovery of normal LV size and function in $22 \%$ of patients [5]. One explanation for this phenomenon might be related to our findings of a less remodelled myocardium which inherits a higher chance for recovery. These results can be supported by the study of Masci et al. who found in adult patients with idiopathic DCM, that the absence of LGE was a strong independent predictor of LV reverse remodelling at 2years follow-up independent of LV size and function [13]. Nabeta and colleagues recently demonstrated that the estimation of fibrosis by baseline CMR-LGE is superior in predicting reverse remodelling and outcome than the estimation of fibrosis by baseline endomyocardial biopsy [41]. Due to the high number of patients in our study cohort who underwent pulmonary artery banding or cardiac transplantation, procedures that affect the natural history of this disease, only a minority of patients received exclusively medical heart failure therapy not allowing any further statistical analysis of the predictive value of LGE in children with DCM. However, detection of LGE seemed not to have an impact on the occurrence of reverse remodelling or outcome which demonstrates that CMR LGE may not be able to separate those patients with a high chance for recovery from those who will be affected by an aggravation in ventricular function with a subsequent higher risk for death and/or cardiac transplant. Whether myocardial fibrosis in childhood DCM is a factor for evaluating innovative treatment options also taking advantage of the suggested preserved cardiac regenerative capacity of the myocardium of young children will require further investigations [42-44].

\section{Study limitations}

The small study population and the retrospective design of the study were accompanied by a selection bias towards patients presenting in an advanced stage of the disease who were referred for cardiac transplantation might not represent the entire population of children and adolescents with DCM. Therefore, the reported incidence of LGE as well as the observed rate of spontaneous recovery and outcome might differ in reality.

Compared to previous studies, we observed a higher incidence of patients with myocarditis which must be considered when interpreting the data. The reason for this might be related to the fact that other causes for DCM, especially hereditary forms, were excluded in order to analyse a homogenous patients cohort. While the underlying genetic causes for childhood DCM are increasingly recognized [45], routine genetic testing for mutations known to cause DCM is not performed at our institution which may have resulted in a certain selection bias.

Identification of LGE in very young patients may be limited by respiratory or motion artifacts related to spontaneous breathing under sedation. Furthermore, spatial resolution of the CMR sequence might be too low to detect small areas of local fibrosis in neonates and infants thereby underestimating its real prevalence. However, in a previous study in neonates and infants with ischemic DCM [21], LGE could be reliably assessed. Because no additional method besides LGE was used to confirm histologically proven areas of myocardial fibrosis thereby potentially missing important signs of adverse ventricular remodelling. Furthermore, the used LGE technique cannot detect perivascular and interstitial myocardial fibrosis that is frequently observed in non-ischemic DCM [46]. Further developments of T1 mapping sequences that enable reliable quantification of the extracellular space in younger patients may potentially provide further and earlier insights into adverse cardiac remodelling in pediatric heart failure.

\section{Conclusions}

In children and adolescents with DCM, focal histologically proven myocardial fibrosis is rarely detected by LGE CMR despite marked LV dilatation and severely depressed LV function. LGE occurred in various patterns and mostly in patients with inflammatory cardiomyopathy. It remains unclear whether myocardial fibrosis in childhood DCM reflects different endogenous repair mechanisms that enable favourable reverse remodelling. Despite the small study population, our findings suggest that LGE may not 
be able to predict ventricular recovery or allowing reliable risk stratification as seen in adult patients. Nevertheless, the observed differences in incidence of myocardial fibrosis compared to adult patients may influence the therapeutic strategy in childhood DCM taking advantage of a less remodelled LV.

\section{Abbreviations}

ACE-I: Angiotensin-converting inhibitors; ARB: Angiotensin receptor blocker; BNP: B-type natriuretic peptide; BSA: Body surface area; CD: Cluster of differentiation; CMR: Cardiovascular magnetic resonance; DCM: Dilated cardiomyopathy; EDV/ESV/SV: Enddiastolic/endsystolic/stroke volume; EF: Ejection fraction; HHV: Human herpes virus; HLA: Human leucocyte antigen; LGE: Late gadolinium enhancement; LVEDD: Left ventricular end-diastolic diameter; LV: Left ventricle; NYHA: New York Heart Association; PAB: Pulmonary artery banding; PVB 19: Parvovirus B19; RV: Right ventricle; TNI: Troponin I.

\section{Competing interests}

The authors declare that they have no competing interests.

\section{Authors' contributions}

$\mathrm{HL}, \mathrm{KK}, \mathrm{RK}, \mathrm{DS}$ and CA have contributed to the conception and design of the study.

$H L, K G, M K, A M, N M$ and KK have contributed to the acquisition of data, analysis and interpretation of the data.

$\mathrm{KK}, J \mathrm{~B}, \mathrm{RK}, \mathrm{DS}$ and CA have contributed to the drafting of the article and have revised it critically for important intellectual content.

\section{Acknowledgements}

This study was supported by the Willy Robert Pitzer Stiftung, Bad Nauheim, Germany.

\section{Author details}

${ }^{1}$ Pediatric Heart Centre, University Children's Hospital Giessen, Feulgenstr. 12, D-35392 Giessen, Germany. ${ }^{2}$ Department of Molecular Pathology, University Hospital Tübingen, Tübingen, Germany.

\section{Received: 30 January 2015 Accepted: 1 May 2015}

\section{Published online: 15 May 2015}

\section{References}

1. Kantor PF, Abraham JR, Dipchand Al, Benson LN, Redington AN. The impact of changing medical therapy on transplantation-free survival in pediatric dilated cardiomyopathy. J Am Coll Cardiol. 2010;55:1377-84.

2. Towbin JA, Lowe AM, Colan SD, Sleeper LA, Orav EJ, Clunie S, et al. Incidence, causes, and outcomes of dilated cardiomyopathy in children. JAMA : the journal of the American Medical Association. 2006;296:1867-76.

3. Tsirka AE, Trinkaus K, Chen SC, Lipshultz SE, Towbin JA, Colan SD, et al. Improved outcomes of pediatric dilated cardiomyopathy with utilization of heart transplantation. J Am Coll Cardiol. 2004;44:391-7.

4. Foerster SR, Canter CE, Cinar A, Sleeper LA, Webber SA, Pahl E, et al. Ventricular remodeling and survival are more favorable for myocarditis than for idiopathic dilated cardiomyopathy in childhood: an outcomes study from the Pediatric Cardiomyopathy Registry. Circulation Heart failure. 2010;3:689-97.

5. Everitt MD, Sleeper LA, Lu M, Canter CE, Pahl E, Wilkinson JD, Addonizio L, Towbin JA, Rossano J, Singh RK, et al: Recovery of Echocardiographic Function in Children with Idiopathic Dilated Cardiomyopathy: Results from the Pediatric Cardiomyopathy Registry. Journal of the American College of Cardiology 2014.

6. O'Sullivan JJ, Roche SL, Crossland DS, Chaudhari MP, Kirk RC, Asif H. Recovery of heart function in children with acute severe heart failure. Transplantation. 2008:85:975-9.

7. Alvarez JA, Orav EJ, Wilkinson JD, Fleming $L E$, Lee DJ, Sleeper $L A$, et al. Competing risks for death and cardiac transplantation in children with dilated cardiomyopathy: results from the pediatric cardiomyopathy registry. Circulation. 2011:124:814-23.

8. Daubeney PE, Nugent AW, Chondros P, Carlin JB, Colan SD, Cheung M, et al. Clinical features and outcomes of childhood dilated cardiomyopathy: results from a national population-based study. Circulation. 2006;114:2671-8.
9. Wu KC, Weiss RG, Thiemann DR, Kitagawa K, Schmidt A, Dalal D, et al. Late gadolinium enhancement by cardiovascular magnetic resonance heralds an adverse prognosis in nonischemic cardiomyopathy. J Am Coll Cardiol. 2008:51:2414-21.

10. Assomull RG, Prasad SK, Lyne J, Smith G, Burman ED, Khan M, et al. Cardiovascular magnetic resonance, fibrosis, and prognosis in dilated cardiomyopathy. J Am Coll Cardiol. 2006;48:1977-85.

11. Gulati A, Jabbour A, Ismail TF, Guha K, Khwaja J, Raza S, et al. Association of fibrosis with mortality and sudden cardiac death in patients with nonischemic dilated cardiomyopathy. JAMA : the journal of the American Medical Association. 2013;309:896-908.

12. Lehrke $\mathrm{S}$, Lossnitzer D, Schob M, Steen H, Merten C, Kemmling H, et al. Use of cardiovascular magnetic resonance for risk stratification in chronic heart failure: prognostic value of late gadolinium enhancement in patients with non-ischaemic dilated cardiomyopathy. Heart. 2011;97:727-32.

13. Masci PG, Schuurman R, Andrea B, Ripoli A, Coceani M, Chiappino S, et al. Myocardial fibrosis as a key determinant of left ventricular remodeling in idiopathic dilated cardiomyopathy: a contrast-enhanced cardiovascular magnetic study. Circulation Cardiovascular imaging. 2013;6:790-9.

14. Babu-Narayan SV, Kilner PJ, Li W, Moon JC, Goktekin O, Davlouros PA, et al. Ventricular fibrosis suggested by cardiovascular magnetic resonance in adults with repaired tetralogy of fallot and its relationship to adverse markers of clinical outcome. Circulation. 2006;113:405-13.

15. Babu-Narayan SV, Goktekin O, Moon JC, Broberg CS, Pantely GA, Pennell DJ, et al. Late gadolinium enhancement cardiovascular magnetic resonance of the systemic right ventricle in adults with previous atrial redirection surgery for transposition of the great arteries. Circulation. 2005;111:2091-8.

16. Smith BM, Dorfman AL, Yu S, Russell MW, Agarwal PP, Mahani MG, et al. Clinical significance of late gadolinium enhancement in patients $<20$ years of age with hypertrophic cardiomyopathy. Am J Cardiol. 2014;113:1234-9.

17. Mahrholdt H, Goedecke C, Wagner A, Meinhardt G, Athanasiadis A, Vogelsberg $\mathrm{H}$, et al. Cardiovascular magnetic resonance assessment of human myocarditis: a comparison to histology and molecular pathology. Circulation. 2004;109:1250-8.

18. Kindermann I, Kindermann M, Kandolf R, Klingel K, Bultmann B, Muller T, et al. Predictors of outcome in patients with suspected myocarditis. Circulation. 2008;1 18:639-48.

19. Schranz D, Rupp S, Muller M, Schmidt D, Bauer A, Valeske K, et al. Pulmonary artery banding in infants and young children with left ventricular dilated cardiomyopathy: A novel therapeutic strategy before heart transplantation. The Journal of heart and lung transplantation : the official publication of the International Society for Heart Transplantation. 2013:32:475-81.

20. Perazzolo Marra M, De Lazzari M, Zorzi A, Migliore F, Zilio F, Calore C, et al. Impact of the presence and amount of myocardial fibrosis by cardiac magnetic resonance on arrhythmic outcome and sudden cardiac death in nonischemic dilated cardiomyopathy. Heart rhythm : the official journal of the Heart Rhythm Society. 2014;11:856-63.

21. Latus H, Gummel K, Rupp S, Mueller M, Jux C, Kerst G, et al. Cardiovascular magnetic resonance assessment of ventricular function and myocardial scarring before and early after repair of anomalous left coronary artery from the pulmonary artery. Journal of cardiovascular magnetic resonance : official journal of the Society for Cardiovascular Magnetic Resonance. 2014;16:3.

22. Alkon J, Friedberg MK, Manlhiot C, Manickaraj AK, Kinnear C, McCrindle BW, et al. Genetic variations in hypoxia response genes influence hypertrophic cardiomyopathy phenotype. Pediatr Res. 2012;72:583-92.

23. Jeewa A, Manickaraj AK, Mertens L, Manlhiot C, Kinnear C, Mondal T, et al. Genetic determinants of right-ventricular remodeling after tetralogy of Fallot repair. Pediatr Res. 2012;72:407-13.

24. Grosse-Wortmann L, Roche SL, Yoo SJ, Seed M, Kantor P. Early changes in right ventricular function and their clinical consequences in childhood and adolescent dilated cardiomyopathy. Cardiol Young. 2010;20:418-25.

25. McCrohon JA, Moon JC, Prasad SK, McKenna WJ, Lorenz CH, Coats AJ, et al. Differentiation of heart failure related to dilated cardiomyopathy and coronary artery disease using gadolinium-enhanced cardiovascular magnetic resonance. Circulation. 2003;108:54-9.

26. Almehmadi F, Joncas SX, Nevis I, Zahrani M, Bokhari M, Stirrat J, Yee R, Fine NM, White JA: Prevalence of Myocardial Fibrosis Patterns in Patients with Systolic Dysfunction: Prognostic Significance for the Prediction of Sudden Cardiac Arrest or Appropriate ICD Therapy. Circulation Cardiovascular imaging 2014. 
27. Mahrholdt H, Wagner A, Judd RM, Sechtem U, Kim RJ. Delayed enhancement cardiovascular magnetic resonance assessment of non-ischaemic cardiomyopathies. Eur Heart J. 2005;26:1461-74.

28. Grun S, Schumm J, Greulich S, Wagner A, Schneider S, Bruder O, et al. Long-term follow-up of biopsy-proven viral myocarditis: predictors of mortality and incomplete recovery. J Am Coll Cardiol. 2012;59:1604-15.

29. Mahrholdt H, Wagner A, Deluigi CC, Kispert E, Hager S, Meinhardt G, et al. Presentation, patterns of myocardial damage, and clinical course of viral myocarditis. Circulation. 2006;114:1581-90.

30. Ryoke T, Gu Y, Mao L, Hongo M, Clark RG, Peterson KL, et al. Progressive cardiac dysfunction and fibrosis in the cardiomyopathic hamster and effects of growth hormone and angiotensin-converting enzyme inhibition. Circulation. 1999;100:1734-43.

31. Baig MK, Mahon N, McKenna WJ, Caforio AL, Bonow RO, Francis GS, et al. The pathophysiology of advanced heart failure. Am Heart J. 1998;135:S216-230.

32. Mann DL, Bristow MR. Mechanisms and models in heart failure: the biomechanical model and beyond. Circulation. 2005;111:2837-49.

33. Schalla S, Bekkers SC, Dennert R, van Suylen RJ, Waltenberger J, Leiner T, et al. Replacement and reactive myocardial fibrosis in idiopathic dilated cardiomyopathy: comparison of magnetic resonance imaging with right ventricular biopsy. Eur J Heart Fail. 2010;12:227-31.

34. Nishikawa T, Uto K, Kanai S, Oda H, Kawamura S, Nakanishi T, et al. Histopathological aspects of cardiac biopsy in pediatric patients with dilated cardiomyopathy. Pediatrics international : official journal of the Japan Pediatric Society. 2011;53:350-3

35. Crossland DS, Edmonds K, Rassl D, Black F, Dark JH, Smith J, et al. Histology of the explanted hearts of children transplanted for dilated cardiomyopathy. Pediatr Transplant. 2008;12:85-90.

36. Nishikawa T, Ishiyama S, Sakomura Y, Nakazawa M, Momma K, Hiroe M, et al. Ultrastructural features of the myocardium of children with dilated cardiomyopathy. Heart Vessel. 1999;14:52-6.

37. Hsia TY, Ringewald JM, Stroud RE, Forbus GA, Bradley SM, Chung WK, et al. Determinants of extracellular matrix remodelling are differentially expressed in paediatric and adult dilated cardiomyopathy. Eur J Heart Fail. 2011;13:271-7.

38. Miyamoto SD, Stauffer BL, Nakano S, Sobus R, Nunley K. Nelson P. Beta-adrenergic adaptation in paediatric idiopathic dilated cardiomyopathy. European heart journal: Sucharov CC; 2012.

39. Alexander PM, Daubeney PE, Nugent AW, Lee KJ, Turner C, Colan SD, et al. Long-term outcomes of dilated cardiomyopathy diagnosed during childhood: results from a national population-based study of childhood cardiomyopathy. Circulation. 2013;128:2039-46.

40. Matsumura Y, Hoshikawa-Nagai E, Kubo T, Yamasaki N, Furuno T, Kitaoka H, et al. Left ventricular reverse remodeling in long-term ( $>12$ years) survivors with idiopathic dilated cardiomyopathy. Am J Cardiol. 2013;111:106-10.

41. Nabeta $T$, Inomata $T$, lida $Y$, Ikeda $Y$, Iwamoto M, Ishii S, Sato T, Watanabe I, Naruke T, Shinagawa $H$, et al: Baseline cardiac magnetic resonance imaging versus baseline endomyocardial biopsy for the prediction of left ventricula reverse remodeling and prognosis in response to therapy in patients with idiopathic dilated cardiomyopathy. Heart and vessels 2013.

42. Mishra R, Vijayan K, Colletti EJ, Harrington DA, Matthiesen TS, Simpson D, et al. Characterization and functionality of cardiac progenitor cells in congenital heart patients. Circulation. 2011;123:364-73.

43. Amir G, Ma X, Reddy VM, Hanley FL, Reinhartz O, Ramamoorthy C, et al. Dynamics of human myocardial progenitor cell populations in the neonatal period. Ann Thorac Surg. 2008;86:1311-9.

44. Porrello ER, Mahmoud Al, Simpson E, Hill JA, Richardson JA, Olson EN, et al. Transient regenerative potential of the neonatal mouse heart. Science. 2011;331:1078-80.

45. Dhandapany PS, Razzaque MA, Muthusami U, Kunnoth S, Edwards JJ, Mulero-Navarro S, et al. RAF1 mutations in childhood-onset dilated cardiomyopathy. Nat Genet. 2014;46:635-9.

46. Sibley CT, Noureldin RA, Gai N, Nacif MS, Liu S, Turkbey EB, et al. T1 Mapping in cardiomyopathy at cardiac MR: comparison with endomyocardial biopsy. Radiology. 2012;265:724-32.

\section{Submit your next manuscript to BioMed Central and take full advantage of:}

- Convenient online submission

- Thorough peer review

- No space constraints or color figure charges

- Immediate publication on acceptance

- Inclusion in PubMed, CAS, Scopus and Google Scholar

- Research which is freely available for redistribution

Submit your manuscript at www.biomedcentral.com/submit 